Archived version from NCDOCKS Institutional Repository http://libres.uncg.edu/ir/asu/

\title{
Appalachľăn
}

$\overline{\text { B O O N E, N O R T H C A R O L I N A }}$

\section{Effects of a Flavonoid-Rich Juice on Inflammation, Oxidative Stress, and Immunity in Elite Swimmers: A Metabolomics- Based Approach}

\author{
Authors: \\ Amy M. Knab, David C. Nieman, Nicholas D. Gillitt, R. Andrew Shanely, \\ Lynn Cialdella-Kam, Dru A. Henson, and Wei Sha
}

\begin{abstract}
The effects of a flavonoid-rich fresh fruit and vegetable juice (JUICE) on chronic resting and postexercise inflammation, oxidative stress, immune function, and metabolic profiles (metabolomics analysis, gas-chromatography mass-spectrometry platform) in elite sprint and middle-distance swimmers were studied. In a randomized, crossover design with a 3 -wk washout period, swimmers $(n=9)$ completed 10-d training with or without $16 \mathrm{fl} \mathrm{oz}$ of JUICE (230 mg flavonoids) ingested pre- and postworkout. Blood samples were taken presupplementation, post-10-d supplementation, and immediately postexercise, with data analyzed using a $2 \times 3$ repeated-measures ANOVA. Prestudy blood samples were also acquired from nonathletic controls $(\mathrm{n}=7$, age-

and weight-matched) and revealed higher levels of oxidative stress in the swimmers, no differences in inflammation or immune function, and a distinct separation in global metabolic scores (R2Y [cum] $=.971)$. Swim workouts consisted of high-intensity intervals (1:1, 1:2 swim-to-rest ratio) and induced little inflamma-tion, oxidative stress, or immune changes. A distinct separation in global metabolic scores was found pre- to postexercise $(\mathrm{R} 2 \mathrm{Y}[\mathrm{cum}]=.976)$, with shifts detected in a small number of metabolites related to substrate utilization. No effect of 10-d JUICE was found on chronic resting levels or postexercise inflammation, oxidative stress, immune function, and shifts in metabolites. In conclusion, sprint and middle-distance swimmers had a slight chronic elevation in oxidative stress compared with nonathletic controls, experienced a low magnitude of postworkout perturbations in the biomarkers included in this study, and received no apparent benefit other than added nutrient intake from ingesting JUICE pre- and postworkout for 10 days.
\end{abstract} (2013) "Effects of a Flavonoid-Rich Juice on Inflammation, Oxidative Stress, and Immunity in Elite Swimmers: A Metabolomics-Based Approach" International journal of Sport Nutrition and Exercise Metabolism \#23 pp.150-160 Version of Record Available @ (http://www.ncbi.nlm.nih.gov/pubmed/23070789) 


\title{
Effects of a Flavonoid-Rich Juice on Inflammation, Oxidative Stress, and Immunity in Elite Swimmers: A Metabolomics-Based Approach
}

\author{
Amy M. Knab, David C. Nieman, Nicholas D. Gillitt, R. Andrew Shanely, \\ Lynn Cialdella-Kam, Dru A. Henson, and Wei Sha
}

\begin{abstract}
The effects of a flavonoid-rich fresh fruit and vegetable juice (JUICE) on chronic resting and postexercise inflammation, oxidative stress, immune function, and metabolic profiles (metabolomics analysis, gas-chromatography mass-spectrometry platform) in elite sprint and middle-distance swimmers were studied. In a randomized, crossover design with a 3-wk washout period, swimmers $(n=9)$ completed 10-d training with or without $16 \mathrm{fl} \mathrm{oz}$ of JUICE (230 mg flavonoids) ingested pre- and postworkout. Blood samples were taken presupplementation, post-10-d supplementation, and immediately postexercise, with data analyzed using a $2 \times 3$ repeated-measures ANOVA. Prestudy blood samples were also acquired from nonathletic controls $(n=$ 7 , age- and weight-matched) and revealed higher levels of oxidative stress in the swimmers, no differences in inflammation or immune function, and a distinct separation in global metabolic scores $\left(\mathrm{R}^{2} \mathrm{Y}[\mathrm{cum}]=.971\right)$. Swim workouts consisted of high-intensity intervals (1:1, 1:2 swim-to-rest ratio) and induced little inflammation, oxidative stress, or immune changes. A distinct separation in global metabolic scores was found pre- to postexercise $\left(\mathrm{R}^{2} \mathrm{Y}[\mathrm{cum}]=.976\right)$, with shifts detected in a small number of metabolites related to substrate utilization. No effect of 10-d JUICE was found on chronic resting levels or postexercise inflammation, oxidative stress, immune function, and shifts in metabolites. In conclusion, sprint and middle-distance swimmers had a slight chronic elevation in oxidative stress compared with nonathletic controls, experienced a low magnitude of postworkout perturbations in the biomarkers included in this study, and received no apparent benefit other than added nutrient intake from ingesting JUICE pre- and postworkout for 10 days.
\end{abstract}

Keywords: human, athletes, metabolic profiles, cytokines, $\mathrm{F}_{2}$-isoprostanes

Increased consumption of polyphenols in the diet has beneficial health outcomes (Hooper et al., 2008). Flavonoids, the largest class of polyphenols, are plant pigments and signaling molecules, and a high intake is associated with decreased risk of cardiovascular disease (Hooper et al., 2008) and cancer (Neuhouser, 2004). The typical Western diet contains $210 \mathrm{mg} / \mathrm{day}$ (Chun et al., 2010), but this number can be as high at $800 \mathrm{mg} /$ day, largely due to variation in fruit and green-tea consumption. In vitro and animal studies indicate that flavonoids exert anti-inflammatory, antioxidative, antipathogenic, and immunoregulatory influences (Boots et al., 2008; Vrijsen, Everaert, \& Boeye, 1988). Human studies with large doses of flavonoid aglycones have produced disappointing results, but emerging evidence suggests that

Knab, Nieman, Shanely, and Cialdella-Kam are with the Human Performance Laboratory, Appalachian State University; Gillitt, the Dole Nutrition Research Laboratory; and Sha, the Bioinformatics Services Division, University of North Carolina at Charlotte, North Carolina Research Campus, Kannapolis, NC. Henson is with the Dept. of Biology, Appalachian State University, Boone, NC. chronic ingestion of flavonoid-rich fruit or vegetable extracts attenuates exercise-induced immune dysfunction, inflammation, and oxidative stress (Bloomer, Goldfarb, \& McKenzie, 2006; Connolly, McHugh, Padilla-Zakour, Carlson, \& Sayers, 2006; Lyall et al., 2009; Nakazato, Song, \& Waga, 2006; Trombold, Barnes, Critchley, \& Coyle, 2010).

The dosing and time course of flavonoid intervention studies in athletes is quite varied in the literature (Nieman, Stear, Castell, \& Burke, 2010). However, the effectiveness of flavonoids may be increased when they are consumed in combinations (Lila, 2007). The interaction between flavonoids from different subclasses increases their bioavailability and bioactivity (Silberberg et al., 2006). For example, supplementation with a mixture of $1,000 \mathrm{mg}$ of quercetin, $120 \mathrm{mg}$ of epigallocatechin 3-gallate, $400 \mathrm{mg}$ of isoquercetin, and $400 \mathrm{mg}$ of eicosapentaenoic acid and docosahexaenoic acid significantly decreased exerciseinducedinfl and improved innate immune markers compared with quercetin alone (Nieman et al., 2009).

Metabolomics is the measurement of small molecules or metabolites in biological samples. Sports nutrition research is increasingly using metabolomics to augment the use of traditional biomarkers in explaining 
underlying metabolic pathways in nutritional interventions (Evans, DeHaven, Barrett, Mitchell, \& Milgram, 2009; Lee, West, Phillips, \& Britz-McKibbin, 2010; Lewis et al., 2010).

The current study included several unique elements: a supplement of freshly prepared, flavonoid-rich fruit and vegetable juice blend (JUICE); a metabolomics-based approach; and the use of elite swimmers and nonathletic controls. Using a randomized crossover design with a 10-day loading period, we hypothesized that JUICE would counter chronic and acute exercise-induced infl mation, oxidative stress, and immune dysfunction as measured by traditional biomarkers and metabolomics. To improve interpretation of underlying metabolic processes in the comparison between JUICE and control, and for comparison between swimmers and nonathletes, blood samples were analyzed for nontargeted shifts in metabolites using gas-chromatography mass spectrometry.

\section{Methods}

\section{Subjects}

Nine elite (national-level) male sprint and middle-distance swim athletes $(24.6 \pm 0.7$ years $)$ from Team Elite of the SwimMAC Carolina Club were recruited into the study. In addition, 7 nonathletic control subjects, matched for age, gender, and body weight, were recruited for prestudy comparisons of all outcome measures. Control subjects were healthy and nonsmokers and exercised less than $150 \mathrm{~min} /$ week. All study procedures were reviewed and approved by the Appalachian State University institutional review board, and subjects provided informed consent before the initiation of the study.

\section{Study Procedures}

Swimmers were randomized to either JUICE or nonJUICE and drank $8 \mathrm{fl} \mathrm{oz} \mathrm{JUICE} \mathrm{pre-} \mathrm{and} \mathrm{posttraining}$ for 10 consecutive days. A list of JUICE ingredients is outlined in Table 1. The U.S. Department of Agriculture database for the flavonoid content of selected foods was used to estimate the total flavonoid content of $16 \mathrm{fl} \mathrm{oz}$ of JUICE at $230 \mathrm{mg}$. In addition, the Folin-Ciocalteu method was used as a surrogate for total phenolic content (Singleton, Orthofer, \& Lamuela-Raventos, 1999) on a Beckman Coulter DU520 UV/Vis spectrophotometer (Beckman Coulter, Inc.) and revealed that JUICE contained $505 \mathrm{mg}$ gallic-acid equivalent in $16 \mathrm{fl} \mathrm{oz}$. After a 3-week washout period, swimmers crossed over and completed either JUICE or non-JUICE conditions for another 10 days. Athletes were required to continue training as usual during the study, while the control subjects maintained normal eating and physical activity patterns. Control subjects reported for blood draws on the same dates and times prestudy as the swimmers.

Baseline fi testing was conducted 2 weeks before the start of the study for both swimmers and control subjects. Subjects were given food records and instructions on how to complete the 3-day food record, which was collected during the first blood draw. Food records were analyzed using the Food Processor computerized dietary-analysis system (v. 10.4.0, Salem, OR). Baseline fitness tests included aerobic power $\left(\mathrm{VO}_{2 \max }\right)$, anaerobic power, and body composition. For aerobic power, a graded exercise test on a cycle ergometer (Lode Excaliber Sport, Lode B.V., Groningen, Netherlands) with continuous metabolic measurements using the Cosmed Quark CPET metabolic cart (Rome, Italy) was

Table 1 Ingredient List, Flavonoid Content, and Total Phenolics of Juice

\begin{tabular}{lclc}
\hline Ingredient & Amount, $\mathbf{g}$ & Amount, household measure & Flavonoids (mg) \\
\hline Red Delicious apple (including peel) & 78 & $1 / 2$ small apple & 15.62 \\
Orange (including peel) & 52 & $1 / 3$ navel orange & 22.61 \\
Red bell pepper & 51 & $1 / 3$ large pepper & 0.12 \\
Carrot & 26 & $1 / 2$ small carrot $(5$ 1/2" long) & 0.16 \\
Broccoli & 40 & $1 / 2$ cup chopped/diced & 4.78 \\
Parsley & 1 & $1 / 41$ sprigs & 2.33 \\
Tomato & 53 & $1 / 2$ small tomato $\left(21 / 4^{\prime \prime}\right.$ diameter) & 0.78 \\
Cucumber (including peel) & 64 & $1 / 5$ cucumber $\left(81 / 4^{\prime \prime}\right)$ & 0.16 \\
Blueberries & 32 & $1 / 5$ cup (about 25 berries) & 87.87 \\
Strawberries & 31 & 3 medium berries & 10.64 \\
Blackberries & 31 & $1 / 5$ cup of berries & 85.13 \\
Pineapple & 36 & $1 / 5$ cup (chunks) & 0.06 \\
Total flavonoid content & & & \\
Total phenolic content (gallic-acid equivalent) & & & 505 \\
\hline
\end{tabular}

Note. Total mass $=495 \mathrm{~g}(\sim 16 \mathrm{fl} \mathrm{oz})$. 
completed. Body composition was measured using the BodPod system (Life Measurement, Concord, CA). Anaerobic power was measured using the 30-s Wingate test on the Lode cycle ergometer.

Blood samples were collected from overnight-fasted swimmers and controls before each 10-day supplementation period. Swimmers randomized to JUICE consumed $8 \mathrm{fl} \mathrm{oz}$ before and $8 \mathrm{fl} \mathrm{oz}$ after morning workouts for 10 consecutive days. Each workout consisted of a 30- to 45-min warm-up followed by $45 \mathrm{~min}$ of high-intensity interval training (1:1 or 1:2 work-to-rest ratio) and 15-25 min of cooldown swimming (leg kicks and technique drills). Total practice time was $2.0 \mathrm{hr}$ and consisted of approximately 5,500-m swim interval training. Water, food, and other beverages low in polyphenol content were consumed ad libitum during training. The swimmers who participated in this study were sprint or middle-distance national-team-level athletes in a "transition to quality" training phase consisting of lactate-producing workouts to enhance their ability to swim at $\mathrm{VO}_{2 \max }$. This entailed two workouts per day consisting of a combination of swim and resistance training (average 3-3.5 hr/day).

JUICE was prepared fresh daily using a commercialgrade, low-revolution, triturating (removes a portion of the fiber) stainless steel juicer (Angel Juicer 5500, Juicers Australia, Queensland Australia). The swimmers consumed $8 \mathrm{fl} \mathrm{oz} \mathrm{JUICE} \mathrm{pre-} \mathrm{and} \mathrm{postworkout} \mathrm{(total} 16 \mathrm{fl}$ oz/day). Ingredients were purchased daily and kept refrigerated. All food handling and preparation maintained the practices outline in the Sanitation of Food Service Establishments (15A NCAC 18A .2600).

\section{Plasma Cytokines}

Total plasma concentrations of seven inflammatory cytokines (IL-6, TNFa, IFN $\gamma$, IL-1 $\beta$, IL-8, IL-10, and IL-12p70) were determined using an electrochemiluminescence-based solid-phase sandwich immunoassay (Meso Scale Discovery, Gaithersburg, MD). All samples and provided standards were analyzed in duplicate. Pre- and postexercise samples for the cytokines were analyzed on the same assay plate to decrease interkit assay variability.

\section{Oxidative Stress and Antioxidant Capacity}

Plasma $\mathrm{F}_{2}$-isoprostanes were determined using gas-chromatography mass spectrometry (GC-MS; Liu, Morrow, \& Yin, 2009). Plasma was collected from heparinized blood, immediately flash-frozen in liquid nitrogen, and stored at $-80^{\circ} \mathrm{C}$. The samples were used to extract free $\mathrm{F}_{2}$-isoprostanes with deuterated [2 $\mathrm{H} 4$ ] prostaglandin $\mathrm{F}_{2} \alpha$ added as an "internal" standard. The mixture was then added to a C18 Sep Pak column, followed by silica solid-phase extractions. $\mathrm{F}_{2}$-isoprostanes were converted to pentafluorobenzyl esters, subjected to thin-layer chromatography, and converted to trimethylsilyl ether derivatives. Samples were analyzed by a negative-ion chemical ionization GC-MS using an Agilent 6890N gas chromatography interfaced to an Agilent 5975B inert MSD mass spectrometer (Agilent Technologies, Inc., Santa Clara, CA).

Protein carbonyls were measured according to protocol (Cayman Chemical, 10005020). Two hundred twenty $\mu \mathrm{l}$ of sample supernatant were pipetted in duplicate into a microwell plate and read at $370 \mathrm{~nm}$ (Synergy H1 Hybrid Reader, BioTek Instruments Inc., Winooski, VT). Total protein was determined using the duplicate sample aliquot by adding $20 \mu \mathrm{l}$ of sample to the microplate in duplicate followed by $180 \mu \mathrm{l}$ of guanidine hydrochloride and 200 $\mu \mathrm{l}$ of BSA standards and read at 280 and $260 \mathrm{~nm}$.

Total oxygen-radical absorbance capacity (ORAC) was measured using methods described previously (Huang, Ou, Hampsch-Woodill, Flanagan, \& Prior, 2002; Ou, Hampsch-Woodill, \& Prior, 2001; Prior et al., 2003). Blanks, Trolox standards $(25 \mu \mathrm{l})$, and plasma samples from EDTA-treated blood were loaded into appropriate microtiter plate wells, followed by fluorescein working solution $(100 \mu \mathrm{l})$. The plate was then incubated with AAPH working solution $(25 \mu \mathrm{l})$. ORAC values were calculated by a fluorescence plate reader (Synergy H1 Hybrid Reader, BioTek Instruments Inc., Winooski, Vermont) as area under the curve.

\section{Granulocyte and Monocyte Phagocytosis and Oxidative Burst Activity}

Granulocyte phagocytosis (G-PHAG), monocyte phagocytosis (M-PHAG), granulocyte oxidative burst activity (G-OBA), and monocyte oxidative burst activity (M-OBA) were assayed as previously described (Konrad et al., 2011). Briefly, phagocytosis was measured through the uptake of fluorescein-isothiocyanate-labeled Staphylococcus aureus bacteria, and oxidative burst was measured through the oxidation of nonfluorescent hydroethidine to fluorescent ethidium bromide in cells stimulated with unlabeled bacteria. Samples were processed on a Q-Prep workstation (Beckman Coulter, Inc.), and analysis was performed within $18 \mathrm{hr}$ of blood collection using a Beckman Coulter FC10 500 flow cytometer. After the granulocyte and monocyte populations were gated using forward scatter and side scatter, the mean fluorescence intensity (MFI; $\mathrm{x}$-mean) and percent positive cells for fluorescein isothiocyanate and oxidized hydroethidine were determined.

\section{Metabolomics}

Standards (Sigma-Aldrich, St. Louis, MO) representing approximately 257 human metabolites were derivatized and analyzed on an Agilent 7890A GC system coupled to a LECO time-of-flight (TOF) mass analyzer set up for electron ionization. A 3:1 methanol-chloroform solution containing $0.2 \mathrm{mg} / \mathrm{ml} \mathrm{p}$-chlorophenylalanine and heptadecanoic acid was prepared and stored at $-20{ }^{\circ} \mathrm{C}$ until use. The p-chlorophenylalanine and heptadecanoic acid were used as internal standards to check the efficiency 
of the sample preparation process, monitor instrument performance, and correct for instrument variability. For each sample, a 1.05-ml aliquot of chilled 3:1 methanolchloroform solution and 300- $\mu$ l aliquot of serum were added to a chilled GC-MS vial. The samples were vortexed and chilled at $-20{ }^{\circ} \mathrm{C}$ for $10 \mathrm{~min}$, then transferred to individual Eppendorf protein LoBind tubes and centrifuged at $14,000 \mathrm{rpm}$ for $15 \mathrm{~min}$ at $4{ }^{\circ} \mathrm{C}$. Supernatant $(1.00 \mathrm{ml})$ was placed in a clean GC-MS maximum recovery vial and dried under nitrogen. The dried extracts were dissolved in $300 \mu \mathrm{l}$ of $20-\mathrm{mg} / \mathrm{ml}$ methoxyamine in pyridine and shaken at $1,400 \mathrm{rpm}$ for $90 \mathrm{~min}$ at $30{ }^{\circ} \mathrm{C}$. N-methyl-N-(trimethylsilyl)trifluoroacetamide $(300 \mu \mathrm{l})$ was added to each vial and shaken at $1,400 \mathrm{rpm}$ for 60 min at $70{ }^{\circ} \mathrm{C}$. Derivatized extracts were stored at -20 ${ }^{\circ} \mathrm{C}$ for at least $1 \mathrm{hr}$. A $200-\mu \mathrm{l}$ aliquot of supernatant was placed in a deactivated glass insert in a GC-MS vial analyzed by GC-MS.

All samples (both serum extracts and standards for the internal library) were analyzed on an Agilent 7890A GC system coupled to an LECO TOF mass analyzer set up for electron ionization. Sample order for the study samples was randomized. The raw-data files generated by GC-MS were processed using ChromaTOF software (v. 4.42, Leco Co., St. Louis, MO) including baseline denosing, smoothing, peak picking, and peak-signal identification using a metabolite reference file. The minimal signal-to-noise was set to 5 . The resulting data set was organized in a matrix including sample information, signal determination for all the detected peaks (retention time-mass pairs), and an intensity determination for each detected peak. For metabolomics biomarker discovery, the internal analytical quality control criteria/metrics are a percent relative standard deviation $\leq 20 \%$ for study sets containing 100-300 injections. Metabolite annotation was performed by comparing unknown signal patterns from the study samples with those of reference standards in our in-house libraries. These libraries included an internal library of 257 human metabolites established on the GC-TOF system at the David H. Murdock Research Institute. Commercial libraries such as the National Institute of Standards and Technology library 2008 and the LECO/Fiehn Metabolomics Library for GC-MS metabolome data were also used for additional compound annotation. A similarity threshold of $70 \%$ was used for all annotations.

\section{Statistical Analysis}

All data are expressed as $M \pm S E$. The biomarker data were analyzed using a 2 (condition) $\times 3$ (time) repeatedmeasures ANOVA, within-subject design. When interaction effects were significant $(p \leq .05)$, changes between time points within JUICE or water conditions were compared between trials using paired $t$ tests, with significance set after Bonferroni adjustment at $p \leq .025$. For metabolomics analysis, due to the complex study design, several analyses were performed to examine several effects:
1. Time effect in control: A linear model with repeated measures was used to determine if metabolites in control samples (nonathletes) were stable during the four time periods. Control samples were included in this analysis, where metabolite concentration is the response variable, and time (first baseline, 10 days after first baseline, second baseline, 10 days after second baseline) is the predictor variable.

2. Athlete effect: A $t$ test was used to detect metabolomic differences between athletes and nonathletes at baseline.

3. Chronic JUICE effect: A linear mixed model with repeated measures was used to examine the JUICE effect from 10 days JUICE consumption in athletes. In this model, metabolite concentration is the response variable, and treatment (JUICE, water control), time (baseline, preswimming), and Treatment $x$ Time interaction effects were the predictor variables. Metabolites with significant (false-discovery-rate (FDR) -adjusted $p$ value <.05) Treatment $\times$ Time interaction effect were considered to be significantly affected by 10 days JUICE consumption.

4. Swimming effect and JUICE effect during workout acutely: A linear mixed model with repeated measures was used to examine these effects. In this model, metabolite concentration is the response variable, and treatment (JUICE, water control), swimming (preswimming, postswimming), and Treatment $\times$ Swimming interaction effects were predictor variables. Metabolites with signifi (FDR-adjusted $p$ value <.05) Treatment $\times$ Swimming interaction effect were considered to be significantly affected by JUICE consumption during swimming. Comparison between preswimming and postswimming in the non-JUICE group was performed to detect metabolites that were affected by the $2.0-\mathrm{hr}$ workout.

Models in Analyses 3 and 4 also adjust for visit and sequence effect due to the crossover design. All of these analyses were performed in the MIXED procedure in SAS (version 9.2, SAS Institute, Inc., Cary, NC) for each metabolite separately. The Benjamini-Hochberg method for FDR correction in the MULTTEST procedure in SAS was used for multiple testing correction. The normality of the residuals was examined. To improve the normality of the data, the relative concentration of each metabolite was log-transformed, and outliers with Studentized residuals $>3$ or $<-3$ were excluded. Missing values for a given metabolite were imputed with the observed minimum. Partial least-square discriminant analysis (PLS-DA) was used to detect metabolites that can best distinguish the JUICE group from the non-JUICE group before swimming. To eliminate the time effect, for each metabolite, ratio of preswimming to baseline for each subject was calculated and was then used as input data for PLS-DA. Similarly, PLS-DA was used to detect metabolites that can best distinguish the JUICE group from the nonJUICE group during swimming. Ratio of postswimming 
to preswimming for each subject was calculated and was then used as input data for PLS-DA. In these discriminant analyses, the default seven-round cross-validation in the SIMCA-P software package was applied, with $1 / 7$ of the samples being left out of the mathematical model in each round.

\section{Results}

Age was not different between swimmers $(24.6 \pm 0.7$ years) and controls $(25.7 \pm 1.3$ years $)$. Swimmers were significantly fitter $\left(\mathrm{VO}_{2 \max }=53.1 \pm 1.4 \mathrm{ml} \cdot \mathrm{kg}^{-1} \cdot \mathrm{min}^{-1}\right.$, body fat $=11.7 \pm 0.8 \%)$ than controls $\left(\mathrm{VO}_{2 \max }=39.9 \pm\right.$ $2.7 \mathrm{ml} \cdot \mathrm{kg}^{-1} \cdot \mathrm{min}^{-1}$, body fat $=19.6 \pm 1.6 \%$; both $p<$ $.001)$. Dietary analysis showed that swimmers consumed an average of $4,105 \mathrm{kcal} /$ day that consisted of $49 \%$ carbohydrates (510 g/day), $18 \%$ protein (188 g/day), and $33 \%$ fat. Dietary intake met or exceeded all recommended values for macro- and micronutrients.

There were significant differences preintervention between swimmers and control subjects in two measures of oxidative stress (protein carbonyls, $p=.05$, and $\mathrm{F}_{2}$-isoprostanes, $p=.032$ ) and antioxidant capacity (ORAC, $p$ $=.001 ;$ Figure 1$)$. Interaction effects for $\mathrm{F}_{2}$-isoprostanes, protein carbonyls, and ORAC were nonsignificant ( $p=$ $.781, .704, .713$, respectively; Figure 2 ), with small but significant time effects for protein carbonyls and ORAC ( $p=.028, .023$, respectively).

No prestudy measures of inflammation and immune function were different between swimmers and controls (data not shown). The interaction effects for infl cytokines (IFN- $\gamma$, IL-1 $\beta$, IL-6, IL-10, IL-8, IL-12p70, and TNF- $\alpha$ ) were nonsignificant (data not shown). Time effects were significant for IL-6 $(p=.008)$ and IL-10 ( $p$ $=.001$; Table 2 ) but not the other measured cytokines. Interaction effects for the immune measures (G-PHAG, M-PHAG, G-OBA, and M-OBA) were nonsignificant except for M-OBA $(p=.013)$. Time effects were nonsignificant for G-PHAG and M-PHAG $(p=.139, .068$, respectively) but were signifi for G-OBA and $\mathrm{M}-\mathrm{OBA}$ (both $p=.001$ ) [Table 2].

PLS-DA analysis revealed no difference between JUICE and water conditions, and no valid model was found. The GC-MS system detected 325 metabolites, and of these, 257 were annotated using the internal library, with the rest annotated using commercial libraries. Score plots from the PLS-DA model in Figure 3(a) visualized the global metabolic differences between the swimmers and controls, indicating a distinct separation $\left(\mathrm{R}^{2} \mathrm{Y}\right.$ [cum] $=$ $.971)$, with significant shifts in the following metabolites (listed in order of significance): $\mathrm{N}$-acetylglutamine, 3-hydroxymandelic acid, pyruvic acid, dopamine, d-galactose, creatine, beta-alanine, 2,4-dihydroxybutanoic acid, glyceric acid, phenylalanine, fumaric acid, norleucine, and ethanolamine. Score plots from the PLS-DA model in Figure 3(b) visualized the global metabolic differences pre- and postexercise, indicating a distinct separation $\left(\mathrm{R}^{2} \mathrm{Y}\right.$ [cum] $\left.=.976\right)$, with significant shifts in the following metabolites (listed in order of significance): pyruvic acid, propanoic acid, d-fructose, mannose, n-acetylglutamine, norleucine, alloisoleucine, and d-glucuronic acid (FDR <.05).

\section{Discussion}

This study used a metabolomics-based approach to examine the influence of JUICE in elite sprint to middle-distance swim athletes. No effect of JUICE for 10 days was found on chronic or acute (exercise-induced) measures of inflammation, oxidative stress, or immune function. Metabolomics profiling strengthened this interpretation. Oxidative-stress measures were significantly higher and antioxidant capacity lower in the swimmers than in the nonathletic controls, with no difference in inflammatory

Table 2 Inflammation and Immune-Function Markers in the Swimmers Presupplementation, After 10-Day Supplementation (Preexercise Acute), and Postexercise

\begin{tabular}{lccccccc}
\hline & \multicolumn{2}{c}{ Presupplementation } & Postsupplementation & \multicolumn{2}{c}{ Postexercise } & \multirow{p}{*}{ (time, } \\
\cline { 2 - 6 } Marker & Juice & Nonjuice & Juice & Nonjuice & Juice & Nonjuice & interaction) \\
\hline $\begin{array}{l}\text { Inflammation, } \\
\text { pg/ml }\end{array}$ & & & & & & & \\
$\quad$ IL-6 & $0.34 \pm 0.1$ & $0.45 \pm 0.07$ & $0.37 \pm 0.08$ & $0.59 \pm 0.14$ & $0.84 \pm 0.18$ & $0.84 \pm 0.14$ & $.004, .552$ \\
$\quad$ IL-10 & $1.71 \pm 0.34$ & $1.76 \pm 0.29$ & $1.74 \pm 0.33$ & $2.15 \pm 0.36$ & $2.82 \pm 0.40$ & $4.44 \pm 1.04$ & $.001, .347$ \\
Immune function, & & & & & & & \\
MFI & & & & & & & \\
$\quad$ G-PHAG & $110 \pm 10.2$ & $115 \pm 12.0$ & $93.8 \pm 12.9$ & $99.7 \pm 15.9$ & $122 \pm 19.3$ & $118 \pm 16.3$ & $.139, .817$ \\
M-PHAG & $73.1 \pm 6.0$ & $77.5 \pm 7.1$ & $65.7 \pm 9.0$ & $68.7 \pm 9.9$ & $89.6 \pm 14.5$ & $85.9 \pm 10.7$ & $.068, .739$ \\
G-OBA & $12.4 \pm 2.3$ & $10.3 \pm 0.8$ & $12.1 \pm 2.3$ & $9.9 \pm 0.8$ & $13.7 \pm 1.8$ & $12.5 \pm 0.7$ & $.001, .614$ \\
M-OBA & $12.5 \pm 2.2$ & $10.3 \pm 1.0$ & $9.8 \pm 2.2$ & $8.0 \pm 0.7$ & $13.3 \pm 1.8$ & $14.4 \pm 1.6$ & $.001, .013$ \\
\hline
\end{tabular}

Note IL = interleukin; $\mathrm{MFI}=$ mean fluorescence intentensity $; \mathrm{G}=$ granulocyte $; \mathrm{PHAG}=$ phagocytosis; $\mathrm{M}=$ monocyte OBA = oxidative burst activity. 

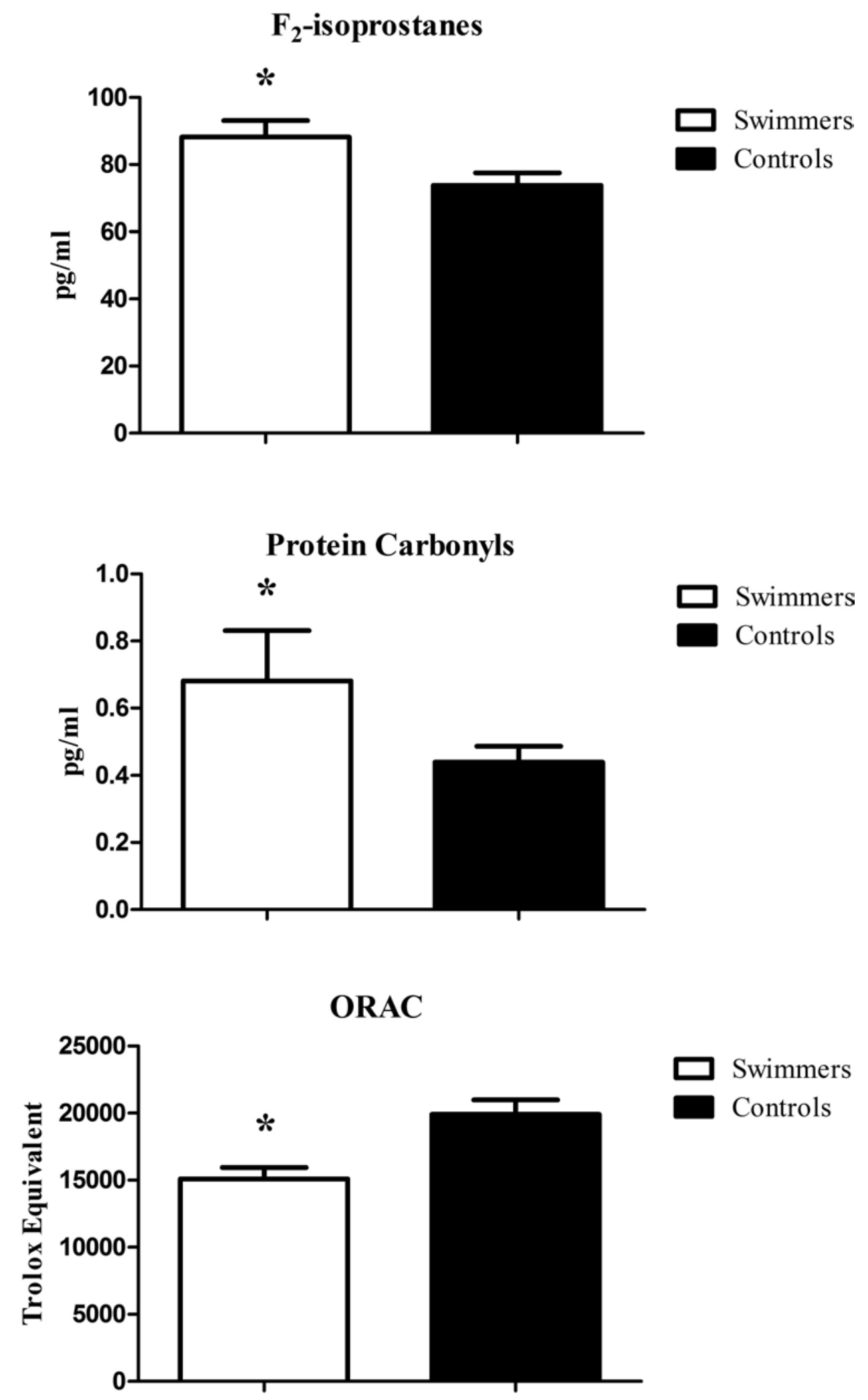

Figure 1 - Differences preintervention between swimmers and control subjects in two measures of oxidative stress $\left(\mathrm{F}_{2}\right.$-isoprostanes, protein carbonyls), and one measure of antioxidant capacity (ORAC). (a) Swimmers had significantly higher levels of $\mathrm{F}_{2}$-isoprostanes $(p=.032)$ than nonathletic controls. (b) Swimmers had significantly higher levels of protein carbonyls $(p=.05)$ than nonathletic controls. (c) Swimmers had significantly lower levels of ORAC $(p=.001)$ than nonathletic controls. 

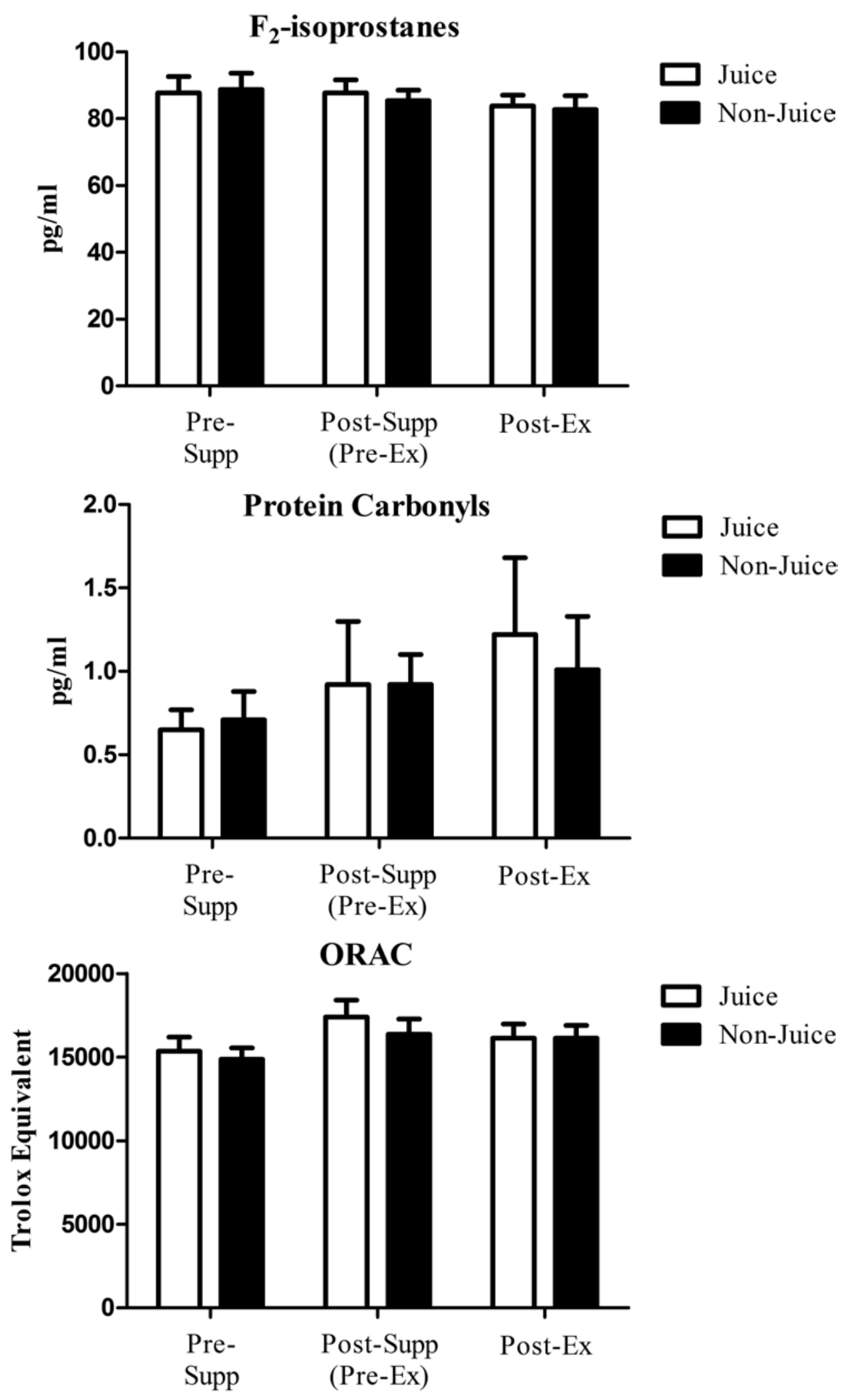

Figure 2 - Measures of $\mathrm{F}_{2}$-isoprostanes, protein carbonyls, and antioxidant capacity (ORAC) in the swimmers pre- and postsupplementation (preexercise) and postexercise. No significant interaction effects (no effect of juice) were found for (a) $F_{2}$-isoprostanes $(p=.781)$, (b) protein carbonyls $(p=.704)$, and (c) ORAC $(p=.713)$. Significant time effects were found for (b) protein carbonyls $(p=.028)$ and (c) ORAC $(p=.023)$. 

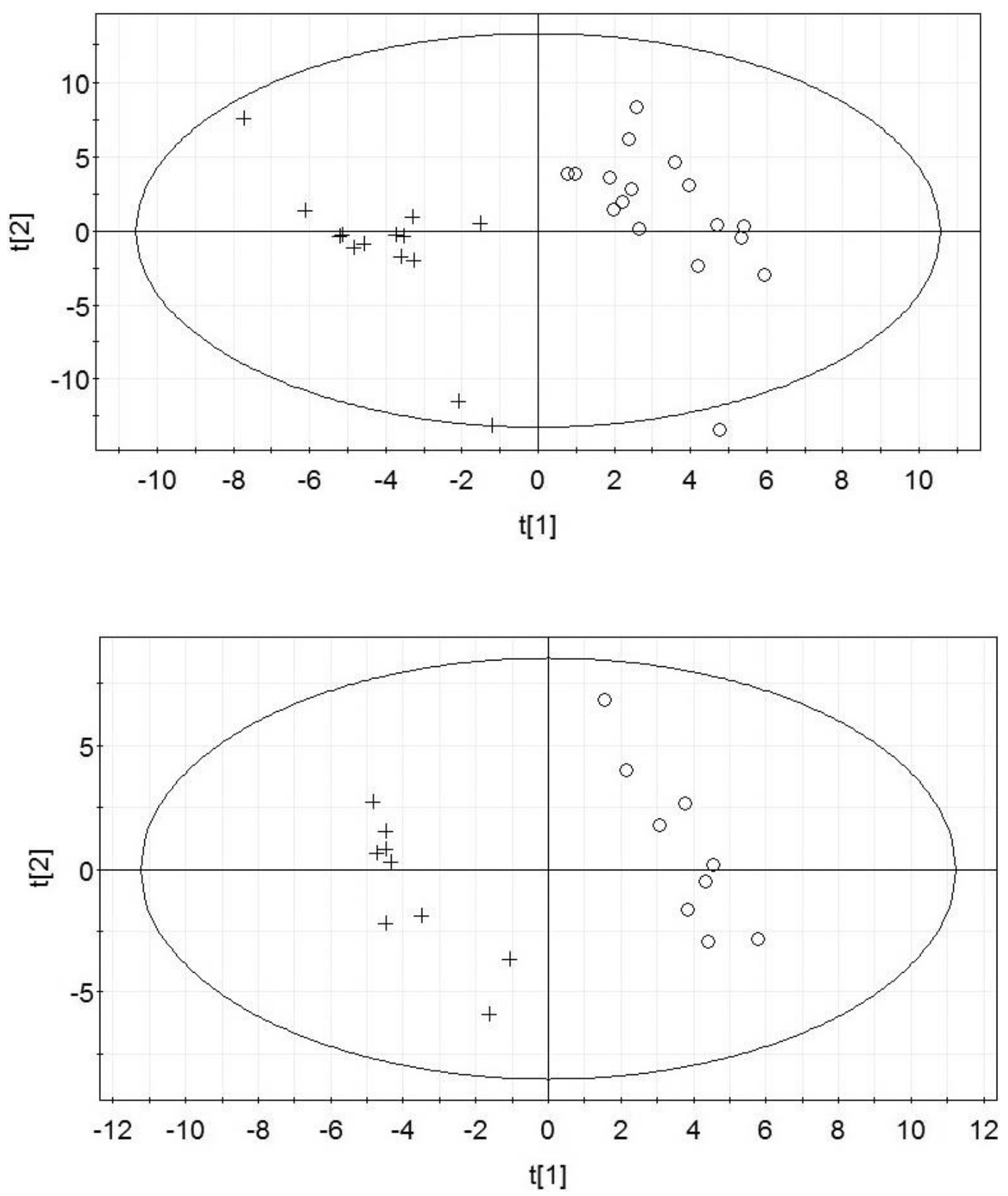

Figure 3 - (a) Partial least-square discriminant analysis (PLS-DA) modeling separated swim athletes from nonathletic controls at baseline samples. $\mathrm{R}^{2} \mathrm{Y}(\mathrm{cum})=.971, \mathrm{Q}^{2} \mathrm{Y}(\mathrm{cum})=.808$. Swimmers represented by circles and nonathletic control represented by plus signs (+). (b) PLS-DA modeling separated preexercise from postexercise in the swimmers. $R^{2} Y($ cum $)=.976, Q^{2} Y($ cum) $=.324$. Preexercise represented by plus signs $(+)$ and postexercise represented by circles. 
markers. A significant difference in metabolic profiles was measured between swimmers and nonathletic controls at baseline. Metabolomics revealed significant metabolite shifts due to acute swim exercise, but the intense workout did not induce signifi oxidative stress or inflammation or alter immune function, except for a positive increase in M-OBA and G-OBA.

JUICE had little room to exert an effect due to low postexercise inflammation and oxidative stress and minimal changes in immune function in the swimmers. Swim training for these athletes involved interval work, allowing swimmers to recover between sets, rather than unrelenting high intensity. Findings from previous studies using athletes with similar training paradigms involving rest intervals (rowers, tennis players, weight lifters) indicate that perturbations in inflammation and immune-function measures are small in contrast to prolonged, intensive running and cycling (Henson et al., 2000). Plasma IL-6 stayed below $1 \mathrm{pg} / \mathrm{ml}$ in the swimmers postworkout, in contrast to far higher levels in runners after a 2 -hr running bout $(\sim 13 \mathrm{pg} / \mathrm{ml})$ or the $160-\mathrm{km}$ Western States Endurance Run ( 78 pg/ml; Nieman et al., 2007). The swim exercise had a small but positive immune effect, as demonstrated with significant increases in M-OBA and G-OBA $(17 \%)$. In contrast, we reported a $38 \%$ and $50 \%$ reduction in G-OBA after a 2-hr intensive treadmill run and the $160-\mathrm{km}$ Western States Endurance Run, respectively (Henson et al., 2008; Konrad et al., 2011)

A literature review by Nieman et al. (2010) showed there is a wide range of dosing regimens being used in nutritional athlete intervention studies. Level of dose (high vs. low) and duration of dosing (time) vary from study to study. In this study we decided to use a 10-day dosing (loading period) because the literature indicates that a 1- to 2-week dosing is sufficient for flavonoid interventions (Lyall et al., 2009). The flavonoid dose of the fruit and vegetable extracts used in those studies is similar to the level in JUICE. JUICE ( $16 \mathrm{fl} \mathrm{oz}$ ) contained a total phenolic content expressed as $505 \mathrm{mg}$ gallic-acid equivalent, or $230 \mathrm{mg}$ of flavonoids. The flavonoid and phenolic dose from $16 \mathrm{fl} \mathrm{oz/day} \mathrm{JUICE,} \mathrm{compared} \mathrm{with}$ the average flavonoid intake of American adults, is a high dietary dose but perhaps somewhat more modest within the context of the swimmer's diet $(4,102 \mathrm{kcal} /$ day). A freeze-dried version of JUICE is currently being tested in cyclists during a 3-day period of intense exercise causing high inflammation, oxidative stress, and immune dysfunction.

The elite sprint swim athletes in this study had slightly elevated preintervention markers of oxidative stress (42\% increased protein carbonyls, $18 \%$ increased $\mathrm{F}_{2}$-isoprostanes) and slightly depressed antioxidant capacity (27\% decreased ORAC) compared with nonathletic but healthy control subjects. This fi is interesting in that we are unaware of any studies that have compared elite swimmers with nonathletes with baseline oxidative-stress markers, but the clinical relevance of these group differences is unclear. The swim training did not acutely affect these oxidative-stress markers, so it is unknown why the swimmers had chronically elevated oxidative-stress markers. Adding to the confusion is that a previous epidemiological study of 1,000 community adults suggested that those in the upper tertile of fitness had decreased $\mathrm{F}_{2}$-isoprostanes (Shanely et al., 2011). The large volume of training experienced by the elite sprint swimmers may have a summation effect over time in amplifying oxidative stress, but this may actually serve a useful purpose in promoting adaptation to training in the context of reactive oxygen species signaling (Powers \& Jackson, 2008).

The metabolomics data from the current study support the null JUICE effects found with the typical biomarkers of inflammation, oxidative stress, and immune function. A significant shift in a small number of metabolites, most related to substrate utilization, was measured after the swim workout. The metabolites that differed between swimmers and controls were mostly related to substrate utilization, as well as a small number likely attributed to supplement use by the swimmers. Our group has previously shown large metabolic shifts related to glutathione production and fuel substrate usage in cyclists after an intense $75-\mathrm{km}$ time trial (Nieman et al., 2012). The interval nature of swim training for these athletes may explain the small but significant metabolite shift postworkout. Another consideration is that the GC-MS platform needs to be combined with the liquid-chromatography MS platform to capture all the metabolite shifts related to both nutrition and exercise interventions (Netzer et al., 2011). Metabolomics also revealed significant differences in a small number of disparate metabolites at baseline between the swim athletes and nonathletic control subjects, but further studies are needed for clarification.

This study was designed to investigate differences in oxidative stress, inflammation, immune function, and metabolic profiles in elite swim athletes and nonathletic control subjects and to investigate the effects of JUICE supplementation in swimmers. Others have shown that 1-2 weeks of fruit and vegetable extracts high in flavonoids are effective in countering exercise-induced inflammation and oxidative stress, in contrast to our findings (Nieman et al., 2010). The JUICE flavonoid dose was similar to that used in other studies (Nieman et al., 2010) and was high enough to significantly alter these biomarkers had they been elevated during training. Swim training did not induce acute changes in inflammation or oxidative stress and mildly enhanced G-OBA and M-OBA. Our findings indicate that sprint and middle-distance swimmers had a slight chronic elevation in oxidative stress but not inflammation compared with nonathletic controls, experienced a low magnitude of postworkout perturbations in measured biomarkers including shifts in metabolites, and received no apparent benefit from ingesting JUICE pre- and postworkout for 10 days. The ingestion of $16 \mathrm{fl} \mathrm{oz/day} \mathrm{of} \mathrm{JUICE} \mathrm{added} \mathrm{significant} \mathrm{nutrients} \mathrm{and}$ flavonoids to the daily diet of these elite swimmers and appears to be the primary benefit experienced. 


\section{Acknowledgments}

We would like to thank Dr. Mary Pat Meaney for her assistance in development and processing of select assays. We are also grateful to SwimMAC Carolina for allowing us access to necessary facilities for JUICE preparation and sample collection. This study was supported through Dole Foods, Inc. No conflicts of interest are reported.

\section{References}

Bloomer, R.J., Goldfarb, A.H., \& McKenzie, M.J. (2006). Oxidative stress response to aerobic exercise: Comparison of antioxidant supplements. Medicine and Science in Sports and Exercise, 38(6), 1098-1105. PubMed doi:10.1249/01. mss.0000222839.51144.3e

Boots, A.W., Wilms, L.C., Swennen, E.L., Kleinjans, J.C., Bast, A., \& Haenen, G.R. (2008). In vitro and ex vivo antiinflammatory activity of quercetin in healthy volunteers. Nutrition (Burbank, Los Angeles County, Calif.), 24(7-8), 703-710. PubMed doi:10.1016/j.nut.2008.03.023

Chun, O.K., Floegel, A., Chung, S.J., Chung, C.E., Song, W.O., \& Koo, S.I. (2010). Estimation of antioxidant intakes from diet and supplements in U.S. adults. The Journal of Nutrition, 140(2), 317-324. PubMed doi:10.3945/ jn.109.114413

Connolly, D.A., McHugh, M.P., Padilla-Zakour, O.I., Carlson, L., \& Sayers, S.P. (2006). Efficacy of a tart cherry juice blend in preventing the symptoms of muscle damage. British Journal of Sports Medicine, 40(8), 679-683, discussion 683. PubMed doi:10.1136/bjsm.2005.025429

Evans, A.M., DeHaven, C.D., Barrett, T., Mitchell, M., \& Milgram, E. (2009). Integrated, nontargeted ultrahigh performance liquid chromatography/electrospray ionization tandem mass spectrometry platform for the identification and relative quantification of the small-molecule complement of biological systems. Analytical Chemistry, 81(16), 6656-6667. PubMed doi:10.1021/ac901536h

Henson, D., Nieman, D.C., Davis, J.M., Dumke, C.L., Gross, S., Murphy, A., . . Mayer, E. (2008). Post-160-km race illness rates and decreases in granulocyte respiratory burst and salivary $\operatorname{IgA}$ output are not countered by quercetin ingestion. International Journal of Sports Medicine, 29, 856-863. PubMed doi:10.1055/s-2007-989424

Henson, D.A., Nieman, D.C., Nehlsen-Cannarella, S.L., Fagoaga, O.R., Shannon, M., Bolton, M.R., ... Schilling, B.K. (2000). Influence of carbohydrate on cytokine and phagocytic responses to $2 \mathrm{~h}$ of rowing. Medicine and Science in Sports and Exercise, 32(8), 1384-1389. PubMed doi:10.1097/00005768-200008000-00005

Hooper, L., Kroon, P.A., Rimm, E.B., Cohn, J.S., Harvey, I., Le Cornu, K.A., . . Cassidy, A. (2008). Flavonoids, fl vonoidrich foods, and cardiovascular risk: A meta-analysis of randomized controlled trials. The American Journal of Clinical Nutrition, 88(1), 38-50. PubMed

Huang, D., Ou, B., Hampsch-Woodill, M., Flanagan, J.A., \& Prior, R.L. (2002). High-throughput assay of oxygen radical absorbance capacity (ORAC) using a multichannel liquid handling system coupled with a microplate fluores- cence reader in 96-well format. Journal of Agricultural and Food Chemistry, 50(16), 4437-4444.

Konrad, M., Nieman, D.C., Henson, D.A., Kennerly, K.M., Jin, F., \& Wallner-Liebmann, S.J. (2011). The acute effect of ingesting a quercetin-based supplement on exerciseinduced inflammation and immune changes in runners. International Journal of Sport Nutrition and Exercise Metabolism, 21(4), 338-346. PubMed

Lee, R., West, D., Phillips, S.M., \& Britz-McKibbin, P. (2010). Differential metabolomics for quantitative assessment of oxidative stress with strenuous exercise and nutritional intervention: Thiol-specific regulation of cellular metabolism with N-acetyl-L-cysteine pretreatment. Analytical Chemistry, 82(7), 2959-2968. PubMed doi:10.1021/ ac9029746

Lewis, G.D., Farrell, L., Wood, M.J., Martinovic, M., Arany, Z., Rowe, G.C., . . . Gerszten, R.E. (2010). Metabolic signatures of exercise in human plasma. Science Translational Medicine, 2(33), 33ra37. PubMed doi:10.1126/ scitranslmed.3001006

Lila, M.A. (2007). From beans to berries and beyond: Teamwork between plant chemicals for protection of optimal human health. Annals of the New York Academy of Sciences, 1114, 372-380. PubMed doi:10.1196/annals.1396.047

Liu, W., Morrow, J.D., \& Yin, H. (2009). Quantification of F2-isoprostanes as a reliable index of oxidative stress in vivo using gas chromatography-mass spectrometry (GC-MS) method. Free Radical Biology \& Medicine, 47(8), 1101-1107. PubMed doi:10.1016/j.freeradbiomed.2009.07.028

Lyall, K.A., Hurst, S.M., Cooney, J., Jensen, D., Lo, K., Hurst, R.D., \& Stevenson, L.M. (2009). Short-term blackcurrant extract consumption modulates exercise-induced oxidative stress and lipopolysaccharide-stimulated inflammatory responses. American Journal of Physiology. Regulatory, Integrative and Comparative Physiology, 297(1), R70R81. PubMed doi:10.1152/ajpregu.90740.2008

Nakazato, K., Song, H., \& Waga, T. (2006). Effects of dietary apple polyphenol on adipose tissues weights in Wistar rats. Experimental Animals, 55(4), 383-389. PubMed doi:10.1538/expanim.55.383

Netzer, M., Weinberger, K.M., Handler, M., Seger, M., Fang, X., Kugler, K.G., . . . Baumgartner, C. (2011). Profiling the human response to physical exercise: A computational strategy for the identification and kinetic analysis of metabolic biomarkers. Journal of Clinical Bioinformatics, 1(1), 34. PubMed doi:10.1186/2043-9113-1-34

Neuhouser, M.L. (2004). Dietary flavonoids and cancer risk: Evidence from human population studies. Nutrition and Cancer, 50(1), 1-7. PubMed doi:10.1207/ s15327914nc5001_1

Nieman, D.C., Gillitt, N.D., Henson, D.A., Sha, W., Shanely, R.A., Knab, A.M., . . . Jin, F. (2012).Bananas as an energy source during exercise: A metabolomics approach. PLoS ONE, 7(5), e37479. PubMed doi:10.1371/journal. pone.0037479

Nieman, D.C., Henson, D.A., Davis, J.M., Dumke, C.L., Gross, S.J., Jenkins, D.P., . . M Mayer, E.P. (2007). Quercetin ingestion does not alter cytokine changes in athletes 
competing in the Western States Endurance Run. Journal of Interferon \& Cytokine Research, 27(12), 1003-1011. PubMed doi:10.1089/jir.2007.0050

Nieman, D.C., Henson, D.A., Maxwell, K.R., Williams, A.S., McAnulty, S.R., Jin, F., . . . Lines, T.C. (2009). Effects of quercetin and EGCG on mitochondrial biogenesis and immunity. Medicine and Science in Sports and Exercise, 41(7), 1467-1475. PubMed doi:10.1249/ MSS.0b013e318199491f

Nieman, D.C., Stear, S.J., Castell, L.M., \& Burke, L.M. (2010). A-Z of nutritional supplements: Dietary supplements, sports nutrition foods and ergogenic aids for health and performance: Part 15. British Journal of Sports Medicine, 44(16), 1202-1205. PubMed doi:10.1136/bjsm.2010.078618

Ou, B., Hampsch-Woodill, M., \& Prior, R.L. (2001). Development and validation of an improved oxygen radical absorbance capacity assay using fluorescein as the fluorescent probe. Journal of Agricultural and Food Chemistry, 49(10), 4619-4626.

Powers, S.K., \& Jackson, M.J. (2008). Exercise-induced oxidative stress: Cellular mechanisms and impact on muscle force production. Physiological Reviews, 88(4), 1243-1276. PubMed doi:10.1152/physrev.00031.2007

Prior, R.L., Hoang, H., Gu, L., Wu, X., Bacchiocca, M., Howard, L., .. . Jacob, R. (2003). Assays for hydrophilic and lipophilic antioxidant capacity (oxygen radical absorbance capacity (ORAC(FL))) of plasma and other biological and food samples. Journal of Agricultural and Food Chemistry, 51(11), 3273-3279.

Shanely, R.A., Nieman, D.C., Henson, D.A., Jin, F., Knab, A.M., \& Sha, W. (2011). Inflammation and oxidative stress are lower in physically fit and active adults. Scandinavian Journal of Medicine \& Science in Sports. Advance online publication. PubMed

Silberberg, M., Morand, C., Mathevon, T., Besson, C., Manach, C., Scalbert, A., \& Remesy, C. (2006). The bioavailability of polyphenols is highly governed by the capacity of the intestine and of the liver to secrete conjugated metabolites. European Journal of Nutrition, 45(2), 88-96. PubMed doi:10.1007/s00394-005-0568-5

Singleton, V.L., Orthofer, R., \& Lamuela-Raventos, R.M. (1999). Analysis of total phenols and other oxidation substrates and antioxidants by means of the folin-ciocalteu reagent. Methods in Enzymology, 299, 152-178. doi:10.1016/S0076-6879(99)99017-1

Trombold, J.R., Barnes, J.N., Critchley, L., \& Coyle, E.F. (2010). Ellagitannin consumption improves strength recovery 2-3 d after eccentric exercise. Medicine and Science in Sports and Exercise, 42(3), 493-498. PubMed doi:10.1249/MSS.0b013e3181b64edd

Vrijsen, R., Everaert, L., \& Boeye, A. (1988). Antiviral activity of flavones and potentiation by ascorbate. The Journal of General Virology, 69(Pt 7), 1749-1751. PubMed doi:10.1099/0022-1317-69-7-1749 